\title{
Multiple Trafficking Signals Regulate Kainate Receptor KA2 Subunit Surface Expression
}

\author{
Zhao Ren, ${ }^{1}$ Nathan J. Riley, ${ }^{1}$ Elizabeth P. Garcia, ${ }^{1}$ James M. Sanders, ${ }^{2}$ Geoffrey T. Swanson, ${ }^{2}$ and John Marshall ${ }^{1}$ \\ ${ }^{1}$ Departments of Molecular Pharmacology, Physiology, and Biotechnology, Brown University, Providence, Rhode Island 02912, and ${ }^{2}$ Department of \\ Pharmacology and Toxicology, University of Texas Medical Branch, Galveston, Texas 77555
}

The kainate receptor subunit KA2 does not form functional homomeric channels despite its structural similarity to the functional glutamate receptor 5-7subunits and high agonist binding affinity in in vitro assays. In this study, we first demonstrate that homomeric KA2 receptors fail to reach the plasma membrane and then identify the molecular mechanisms preventing surface expression. Specifically, we show that KA2 subunits form homooligomeric receptors that are confined to the endoplasmic reticulum (ER). We then demonstrate that, in both heterologous expression systems and primary neurons, the intracellular retention of KA2 is not caused by subunit misfolding but, rather, is mediated through discrete protein trafficking signals, including an arginine-rich ER retention/retrieval motif and a di-leucine endocytic sequence in the $\mathrm{C}$ terminus of the KA2 subunit. Disruption of these motifs results in ER exit and surface expression of KA2 homomeric receptors that remain nonfunctional. Furthermore, our data suggest that the ER retention/retrieval signal in KA2 is sterically shielded during heteromeric assembly, allowing delivery of functional heteromeric receptors to the plasma membrane. Taken together, our results illustrate novel regulatory mechanisms that control the intracellular trafficking and surface expression of kainate receptors.

Key words: kainate receptors; KA2 subunit; intracellular trafficking; ER retention; arginine-rich motif; di-leucine motif; heteromeric assembly

\section{Introduction}

The three types of ionotropic glutamate receptors, NMDA, AMPA, and kainate, are the major mediators of excitatory neurotransmission in the mammalian CNS (Seeburg, 1993; Hollmann and Heinemann, 1994). Studies with recombinant kainate receptors in oocytes and mammalian cells demonstrate that glutamate receptor (GluR) 5-7 subunits alone or in combination can generate functional homomeric or heteromeric receptors (Bettler et al., 1990; Schiffer et al., 1997; Swanson et al., 1997; Cui and Mayer, 1999; Paternain et al., 2000). In contrast, the KA1 and KA2 subunits are functional only when incorporated into heteromeric channels with the GluR5-7 subunits (Wenthold et al., 1994; Schiffer et al., 1997).

The intracellular trafficking and surface delivery of multimeric transmembrane proteins, such as the kainate receptors, are likely to be tightly controlled processes requiring proper folding and assembly of constituent subunits, so that only fully assembled functional receptors can be expressed on the plasma membrane. A number of mechanisms that regulate receptor trafficking have been discovered in recent years; some of which function

\footnotetext{
Received March 21, 2003; revised May 15, 2003; accepted May 19, 2003.

This research was supported by National Institutes of Health Grants NS39309 and NS39063 (J.M.), American Heart Association Grant 9940131 N (J.M.), Centers of Biomedical Research Excellence Grant RR15578 (J.M.), and National Institute of Neurological Disorders and Stroke Grant NS44322 (G.T.S.). We thank Leigh Needleman for providing the hippocampal culture and Dr. Leslie Blair for critical reading of this manuscript.

Correspondence should be addressed to Dr. John Marshall, Department of Molecular Pharmacology, Physiology, and Biotechnology, Brown University, Box G-B4, Providence, RI 02912. E-mail: john_marshall@brown.edu. Copyright $\odot 2003$ Society for Neuroscience $\quad$ 0270-6474/03/236608-09\$15.00/0
}

in early secretory steps, whereas others participate in later events, such as endocytosis and protein degradation. Studies done on immune receptors identified the endoplasmic reticulum (ER) as the primary checkpoint of protein trafficking and revealed a control mechanism for the proper assembly and delivery of multimeric complexes to the plasma membrane (Teasdale and Jackson, 1996). These studies demonstrate that individual subunits contain discrete ER retention motifs and that these signals are masked and disabled in the fully assembled heterooligomeric receptors (Letourneur et al., 1995). Alternatively, unassembled or partially assembled subunits may be retained in the ER because of misfolding and subsequent recognition by chaperone proteins (Kowalski et al., 1998). The ER retention mechanisms can also be coupled to protein degradation processes, adding another level of regulation (Keller et al., 2001).

Recent studies have revealed additional discrete ER retention signals, most of which consist of positively charged residues (e.g., arginine or lysine). For example, a novel RXR (arginine-a large neutral or positively charged residue-arginine) ER retention motif was found in both ion channels and a G-protein-coupled receptor. In the ATP-sensitive potassium channel $\left(\mathrm{K}_{\mathrm{ATP}}\right)$ and $\mathrm{GABA}_{\mathrm{B}}$ receptor, the RXR sequences present in individual subunits are shielded in the mature complexes to enable egress from the ER (Zerangue et al., 1999; Margeta-Mitrovic et al., 2000). The RXR motif is also shown to mediate the ER retention of several splice variants of NMDA receptor subunit NR1 (Standley et al., 2000; Scott et al., 2001). In addition, several anterograde trafficking signals have been identified in potassium channels and 
AMPA receptors, adding another level of complexity to the modulation of protein trafficking (Ma et al., 2001; Stockklausner et al., 2001; Xia et al., 2002).

In the present study, we investigated the assembly-dependent trafficking of the kainate receptor subunit KA2. We now report that KA2 subunits are retained in the ER despite their ability to assemble into homooligomeric receptors. The intracellular retention is not initiated by subunit misfolding but, rather, is mediated through discrete protein trafficking signals, namely an arginine-rich motif and a di-leucine sequence in the C-terminal tail of KA2. Disruption of these motifs results in subsequent ER exit and surface expression of KA2 homomeric receptors. During heteromeric assembly, these trafficking signals may be sterically masked, allowing delivery of functional heteromeric receptors to the plasma membrane. Interestingly, surface-expressed mutant KA2 homomeric receptors remain nonresponsive to agonists, indicating that only fully assembled heteromeric KA2-containing receptors are functional, and their efficient assembly and surface expression are ensured by multiple trafficking checkpoints along the secretory pathway.

\section{Materials and Methods}

Cell culture. Human embryonic kidney 293 (HEK293) cells, normal rat kidney (NRK) cells, and COS-7 cells [American Type Culture Collection (ATCC), Manassas, VA] were grown in DMEM (Invitrogen, Gaithersburg, MD) supplemented with $10 \%$ FBS and $50 \mathrm{U} / \mathrm{ml}$ penicillin and streptomycin $\left(37^{\circ} \mathrm{C}, 5 \% \mathrm{CO}_{2}\right)$. For immunocytochemistry experiments, cells were plated onto poly-D-lysine-coated glass coverslips in the same growth medium. Primary cultures of hippocampal neurons were obtained from embryonic day 18 (E18) rat embryos. Briefly, the hippocampus was dissected from E18 Sprague Dawley rats and dissociated with trypsin. Dissociated cells were then plated onto glass coverslips coated with poly-L-lysine (Sigma, St. Louis, MO) at a density of $7500 \mathrm{cells} / \mathrm{cm}^{2}$ in Neurobasal medium (Invitrogen) supplemented with Glutamax and B27. AraC $(5 \mu \mathrm{M})$ was added $3 \mathrm{~d}$ after plating, and cells were fed twice weekly thereafter.

Molecular biology. Primers containing coding sequences for hemagglutinin (HA; YPYDVPDYA) or FLAG (DYKDDDDK) were used to amplify the KA2 C terminus by PCR; both PCR fragments were then digested with EcoRI and $X b a I$, and used to replace the corresponding piece in KA2-pcDNA3 expression vector. Both tags were inserted after the last residue of KA2. A sequence containing five myc (MEQKLISEEDLNE) epitopes in tandem was amplified by PCR from a pCS2+MT vector and inserted after residue 14 (counted from the initiator methionine) of KA2, using an NruI site created by QuikChange (Stratagene, La Jolla, CA). The green fluorescent protein (GFP)-tagged GluR6-pcDNA3 construct was a generous gift from Dr. Steve Heinemann (The Salk Institute, La Jolla, CA). An EcoRI site was engineered into the beginning of the GluR6 C terminus by QuikChange to facilitate the generation of chimeras. Two chimeras, GFP-GluR6/KA2 (GFP-tagged GluR6 with the C terminus of $\mathrm{KA2}$ ) and myc-KA2/G6 (myc-tagged KA2 with the C terminus of GluR6), were generated by a PCR method. The C termini of GluR6 and KA2 were amplified by PCR, and both fragments were then digested with $E c o R I$ and $X b a I$ and used to replace their counterparts in the respective expression vectors. Another KA2 chimera (named myc-KA2-G6C), with an appended C terminus from GluR6, was generated with a two-stage PCR method, using two sets of overlapping primers that also code a six-glycine linker. The PCR fragment containing both C termini in tandem was then digested with EcoRI and $\mathrm{XbaI}$ and used to replace the corresponding piece in KA2-pcDNA3. The pMLSV N1/N4 expression vector for human Tac was purchased from ATCC. The Tac coding sequence was then amplified by PCR and subcloned into a HindIII-XbaI site in pcDNA3. To generate the Tac-KA2 chimera, we first amplified the $\mathrm{N}$-terminal and transmembrane sequences of Tac, as well as the $\mathrm{C}$ terminus of KA2 by PCR, and then ligated both fragments into a HindIII-XbaI site in pcDNA3 after digestion. By taking advantage of an endogenous EcoRI site at the beginning of the KA2 C-terminal coding sequence, no extra residues were inserted. Deletion mutants of Tac-KA2 were made by PCR amplification of the desired sequences and subsequent subcloning into the HindIII-XbaI site in the pcDNA3 expression vector. The R850$854 \mathrm{~A}$ mutation in both KA2 and Tac-KA2 was generated through the two-stage PCR method, using two sets of overlapping primers that code the desired mutation. The PCR fragment containing the desired mutation was digested with EcoRI and $\mathrm{XbaI}$ and used to replace the corresponding piece coding the wild-type sequence. The Tac-KA2 (T858S859DD) mutant was made with the same method. The Tac-KA2 and KA2 double mutants (R862-866A and LL874-875VV or LL908-909VV) were also made with a similar method, using the R862-866A mutant as a template. The pEGFP-C2 and pDsRed2-ER vectors were purchased from BD Biosciences (San Jose, CA). A GFP-GluR6C fusion protein was generated by PCR amplification of the C-terminal sequence and subsequent insertion into an EcoRI-XbaI site in pEGFP-C2. GluR5-pRK5 and postsynaptic density (PSD)-95pGW vectors were gifts from Dr. Peter Seeburg (Max-Planck Institute for Medical Research, Heidelberg, Germany) and Dr. Morgan Sheng (Massachusetts Institute of Technology, Cambridge, MA), respectively. All the inserted oligonucleotides and PCR-derived sequences were entirely sequenced.

Transfection. HEK293, COS-7, and NRK cells were transfected using the Fugene 6 transfection reagent (Roche Molecular Biochemicals, Indianapolis, IN), following the manufacturer's recommended protocol for transient transfection of adherent cells. Cultured hippocampal neurons (in vitro day 7) were transfected with the LipofectAMINE 2000 transfection reagent (Invitrogen), using the manufacturer's recommended protocol. Surface expression and intracellular protein expression were analyzed $48 \mathrm{hr}$ after transfection.

Antibodies. Monoclonal anti-HA antibody (Covance, Princeton, NJ) was used at 1:1000 or 1:150, respectively for Western blots or immunoprecipitation. Monoclonal anti-FLAG (Sigma) was used at $10 \mu \mathrm{g} / \mathrm{ml}$ for Western blots and 1:1000 for immunoprecipitation. Monoclonal antiautofluorescent protein (AFP) (E-6) antibody (Qbiogene, Carlsbad, CA) was used at 1:100 for immunofluorescence. Monoclonal anti-GFP (B-2) antibody (Santa Cruz Biotechnology, Santa Cruz, CA) was used at 1:200 for Western blots. Monoclonal anti-myc (9E10) antibody (Santa Cruz Biotechnology) was used at 1:200 for immunofluorescence and Western blots and 1:1000 for flow cytometry. Polyclonal anti-KA2 and antiGluR6/7 (Upstate, Charlottesville, VA) were used at $9 \mu \mathrm{g} / \mathrm{ml}$ for immunofluorescence and $1 \mu \mathrm{g} / \mathrm{ml}$ for Western blots. Monoclonal anti-Tac (Covance) was used at 1:800 or 1:2000 for immunostaining of heterologous cells or hippocampal neurons and 1:4000 for flow cytometry. Monoclonal anti-PSD-95 (Upstate Biotechnology, Lake Placid, NY), anti-trans-Golgi network (TGN)-38 (Calbiochem, San Diego, CA), antimannosidase II (Covance), and polyclonal anti-giantin (Covance) were used at 1:1000 for immunofluorescence of heterologous cells. Polyclonal anti-calnexin was used at 1:200 for immunostaining. All secondary antibodies conjugated to fluorescein isothiocyanate (FITC), phycoerythrin, rhodamine red-X, or 7-amino-4-methylcoumarin-3-acetic acid (Jackson ImmunoResearch, West Grove, PA) were used at 1:200.

Protein preparation, immunoblotting, and immunoprecipitation. Fortyeight hours after transfection, HEK cells grown in $60 \mathrm{~mm}$ culture dishes were washed once with cold Tris-buffered saline (TBS) and homogenized in $1 \mathrm{ml}$ of lysis buffer (1\% Triton X-100 in TBS with $0.1 \mathrm{~mm}$ PMSF, 10 $\mu \mathrm{g} / \mathrm{ml}$ aprotinin, $10 \mu \mathrm{g} / \mathrm{ml}$ leupeptin, $10 \mu \mathrm{g} / \mathrm{ml}$ pepstatin $\mathrm{A}$, and $2 \mathrm{~mm}$ EDTA, pH 8.0). The samples were then solubilized for $1 \mathrm{hr}$ at $4^{\circ} \mathrm{C}$ and clarified by centrifugation at $14,000 \times g$ for $30 \mathrm{~min}$. The supernatants were then used for immunoblotting and immunoprecipitation experiments. Immunoprecipitations were performed by incubating extracts with $4 \mu \mathrm{g}$ of the appropriate antibodies for $12 \mathrm{hr}$ at $4^{\circ} \mathrm{C}$, followed by incubation with $50 \mu \mathrm{l}$ of a $50 \%$ protein G-Sepharose (Amersham Biosciences, Piscataway, NJ) slurry for $1 \mathrm{hr}$ and four washes in Salter lysis buffer $(0.1 \%$ SDS, $0.5 \%$ deoxycolate, and $1 \%$ NP-40 in TBS with $0.1 \mathrm{~mm}$ PMSF, $10 \mu \mathrm{g} / \mathrm{ml}$ aprotinin, $10 \mu \mathrm{g} / \mathrm{ml}$ leupeptin, $10 \mu \mathrm{g} / \mathrm{ml}$ pepstatin A, and 2 mм EDTA, $\mathrm{pH} 8.0$ ). Bound proteins were eluted from the beads by boiling in $1 \times$ sample buffer and then separated by $8 \%$ SDS-PAGE. Gels were then Western-blotted, immunostained, and visualized with Supersignal West Pico chemiluminescent substrate (Pierce, Rockford, IL).

Biotinylation of cell surface protein. Transfected HEK cells grown in 
poly-D-lysine-coated $60 \mathrm{~mm}$ culture dishes were washed three times with ice-cold PBS and incubated $15 \mathrm{~min}$ with $1.0 \mathrm{mg} / \mathrm{ml} \mathrm{EZ-link} \mathrm{sulfo-} \mathrm{N}$ hydroxysulfosuccinimide (NHS)-S-S-biotin (Pierce) in cold PBS, $\mathrm{pH}$ 8.0 , with gentle agitation at $4^{\circ} \mathrm{C}$. Cells were washed once and incubated with a quenching buffer (192 mm glycine and $25 \mathrm{~mm}$ Tris in PBS) for 10 min. Next, cells were rinsed twice and scraped in cold PBS, pelleted down, and homogenized in lysis buffer as mentioned above. Supernatants were then incubated with $50 \mu \mathrm{l}$ of a $50 \%$ slurry of streptavidinSepharose beads (Amersham Biosciences) for $2 \mathrm{hr}$ at $4^{\circ} \mathrm{C}$. Beads were pelleted by brief centrifugation, and aliquots of the supernatant were taken to represent the unbound intracellular pool. Beads were then washed three times with Salter buffer, and biotinylated proteins were eluted, SDS-PAGEseparated, and immunoblotted as previously described.

Immunofluorescence. For selective labeling of surface receptors, transfected live COS-7 cells were incubated with appropriate primary antibodies (anti-Tac, anti-myc, or anti-AFP) diluted in DMEM supplemented with $5 \% \mathrm{FBS}$ for $1 \mathrm{hr}$ at $4^{\circ} \mathrm{C}$. The cells were then washed with cold PBS and fixed with $4 \%$ paraformaldehyde (PFA) and $4 \%$ sucrose on ice for $20 \mathrm{~min}$. After fixation, the cells were washed with PBS and labeled with fluorescence-conjugated secondary antibodies. Next, cells were permeabilized at room temperature with $0.2 \%$ Triton X-100 for 5 min. Intracellular expression was then determined by sequentially incubating with proper primary antibodies (anti-Tac, anti-GluR6, or anti-KA2) and secondary antibodies for 2 or $1 \mathrm{hr}$, respectively. A slightly modified protocol was used for staining of the transfected hippocampal neurons. Briefly, for surface labeling, cultured live neurons were incubated with anti-Tac diluted in PBS with 5\% normal goat serum (NGS) for $30 \mathrm{~min}$ at room temperature, fixed, and incubated with a rhodamine-conjugated anti-mouse secondary antibody. To detect intracellular expression, neurons were then permeabilized and sequentially incubated with anti-Tac and FITC-conjugated anti-mouse secondary antibody diluted in PBS with $10 \%$ NGS. Both surface and intracellular expression were captured on an Axioskop fluorescence microscope (Zeiss, Thornwood, NY) coupled to a CCD camera (Hamamatsu, Hamamatsu City, Japan) and analyzed by OpenLab imaging software (Improvision, Coventry, UK). Colocalization images were obtained with the permeabilized staining protocol, captured with a Zeiss LSM410 confocal microscope, and analyzed with Renaissance imaging software (Microcosm, Columbia, MD).

Flow-assisted cytometry. Transfected live HEK293 cells grown in sixwell tissue culture plates were incubated with monoclonal anti-Tac or anti-myc antibodies diluted in DMEM supplemented with 5\% serum for $1 \mathrm{hr}$ at $4^{\circ} \mathrm{C}$. Cells were then washed with PBS and incubated with FITC- or phycoerythrin-conjugated anti-mouse secondary antibodies in PBS for 1 hr at $4^{\circ} \mathrm{C}$. After extensive washing, cells were detached from the plates with $500 \mu$ lof PBS plus $5 \mathrm{~mm}$ EDTA and transferred to $12 \times 75 \mathrm{~mm}$ polystyrene test tubes (VWR Scientific, South Plainfield, NJ). Finally, $500 \mu \mathrm{l}$ of $4 \%$ PFA in PBS was added to the tubes to fix the cells. Surface expression was quantified using a FACScalibur cell sorter (BD Biosciences). The background fluorescence was determined using HEK293 cells transfected with Tac or myc-KA2 but incubated without corresponding primary antibodies. Mean fluorescence intensity was acquired and plotted with Origin 5.0 software (Microcal Software, Northampton, MA).

Electrophysiology. HEK293 cells were plated on glass coverslips coated with $100 \mu \mathrm{g} / \mathrm{ml}$ collagen and poly-D-lysine. The following day, the cells were transfected with plasmids containing the myc-KA2 or mutant cDNA alone ( $1.0 \mu \mathrm{g} /$ coverslip) or in combination with GluR5(Q) cDNA [0.5 $\mu \mathrm{g}$ of GluR5 and $1.5 \mu \mathrm{g}$ of myc-KA2 or mutant DNA per coverslip] using the Fugene 6 transfection reagent according to the manufacturer's instructions. A plasmid DNA containing enhanced GFP was included $(0.2 \mu \mathrm{g} /$ coverslip) to allow fluorescent identification of transfected cells. Whole-cell patch-clamp recordings were made with an Axopatch 200B amplifier (Axon Instruments, Foster City, CA) $3 \mathrm{~d}$ after transfection. The external bath solution contained (in $\mathrm{mM}$ ): $150 \mathrm{NaCl}, 2.8 \mathrm{KCl}, 10$ glucose, $2 \mathrm{CaCl}_{2}, 1.0 \mathrm{MgCl}_{2}$, and 10 HEPES, pH adjusted to 7.3 with $\mathrm{NaOH}$. The internal solution was composed of (in mM): $110 \mathrm{CsF}, 30 \mathrm{CsCl}, 4 \mathrm{NaCl}, 0.5$ $\mathrm{CaCl}_{2}, 10$ HEPES, and 5 EGTA, adjusted to $\mathrm{pH} 7.3$ with $\mathrm{CsOH}$. For rapid agonist application exchange, cells were lifted from the coverslip into a laminar solution stream exiting a three-barrel glass pipette (Vitro Dynamics, Rockaway, NJ) pulled to an internal barrel diameter of $\sim 80 \mu \mathrm{m}$

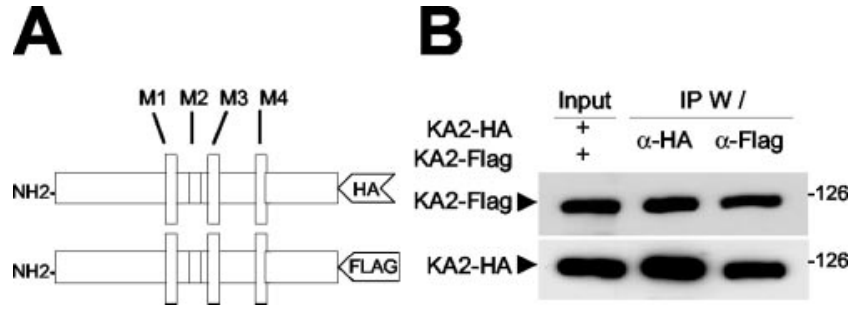

Figure 1. KA2 subunits form homomultimeric receptors in heterologous cells. A, Schematic diagrams of the KA2 constructs used for the experiments. HA and FLAG tags are inserted after the last residue of KA2, and $\mathrm{M} 1-\mathrm{M} 4$ indicate the transmembrane domains. $\mathrm{NH} 2, \mathrm{~N}$ terminus. $B$, KA2-HA and KA2-FLAG form an oligomeric complex in HEK293 cells. HEK293 cells were cotransfected with KA2-HA and KA2-FLAG as indicated. Cell lysate was immunoprecipitated (IP w/) using anti-HA or anti-FLAG antibody, and the precipitates were immunoblotted with anti-FLAG or anti-HA antibody, respectively.

and mounted on a piezo-ceramic bimorph. The solution stream was rapidly moved across the transfected cells by applying voltage to the bimorph with a stimulation-isolation unit triggered by a digital signal from pClamp 8 software. Data was acquired and analyzed using pClamp 8 software (Axon Instruments) and Origin 6.0 (Origin Lab Corp., Northampton, MA).

\section{Results}

The KA2 subunits form homooligomeric receptors that are retained in the ER

Functional ionotropic glutamate receptors are believed to be tetrameric complexes, with each constituent subunit contributing to ligand binding and channel pore formation (Laube et al., 1998; Rosenmund et al., 1998; Bowie and Lange, 2002). Heterologous expression of the KA2 protein does not yield functional channels, which might result from impaired assembly of KA2 subunits into homooligomeric receptors. To address this possibility, we tested whether KA2 subunits with different fusion tags could coimmunoprecipitate with each other when expressed in a heterologous cell
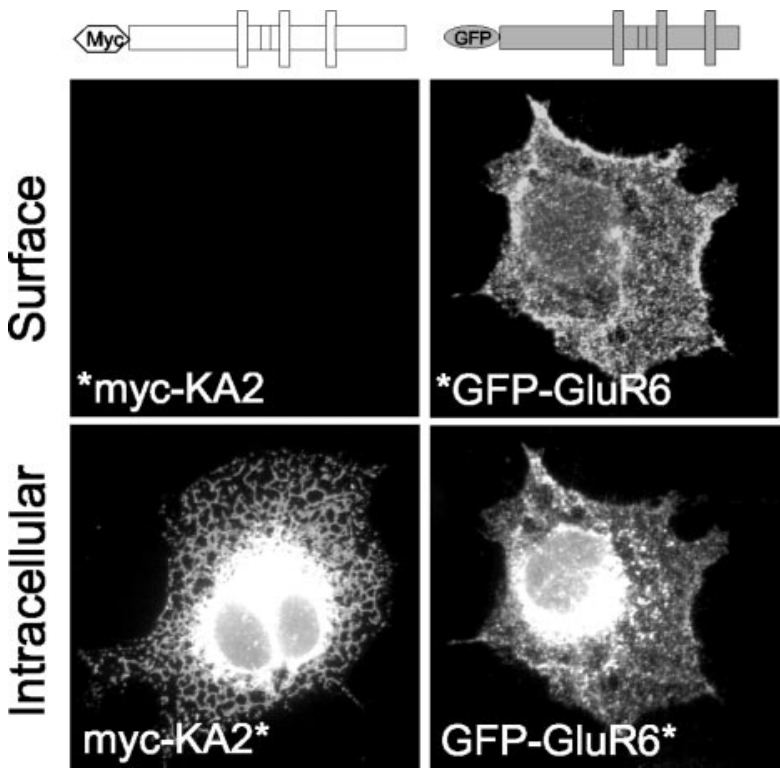

Figure 2. Intracellular retention of KA2 homomeric receptors. Surface and intracellular expression of myc-KA2 and GFP-GluR6 in COS-7 cells is shown. Live cells were labeled with antimyc or anti-AFP antibodies (Surface), permeabilized, and labeled with anti-KA2 or anti-GluR6 antibodies (Intracellular). The constructs used for the staining are illustrated at the top; white bars correspond to KA2 sequences, and gray bars correspond to GluR6 sequences. Asterisks indicate the antibodies used for staining. Note that there is no surface staining for KA2. 


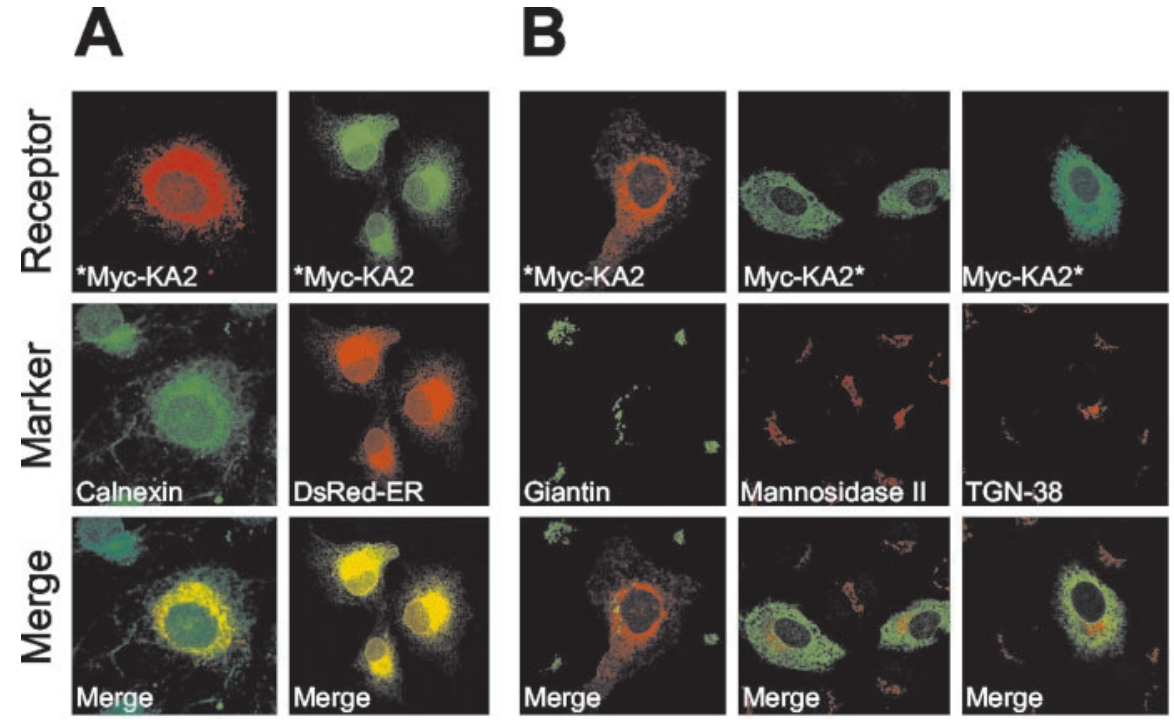

Figure 3. The KA2 homomeric receptors are retained in the ER. $A$, KA2 colocalizes with ER markers. In the left panel, COS-7 cells transfected with myc-KA2 were permeabilized and stained with antibodies against myc (red) and the ER marker calnexin (green); in the right panel, COS-7 cells were cotransfected with myc-KA2 (green) and DsRed-ER (red). Image overlays show extensive colocalization (yellow) in both stains. B, KA2 does not localize to Golgi or tans-Golgi network compartments. COS-7 or NRK cells were transfected with myc-KA2 and costained with the Golgi markers giantin (green), mannosidase II (red), and TGN-38 (red). Image overlays showed no colocalization (yellow) in any stain.

A
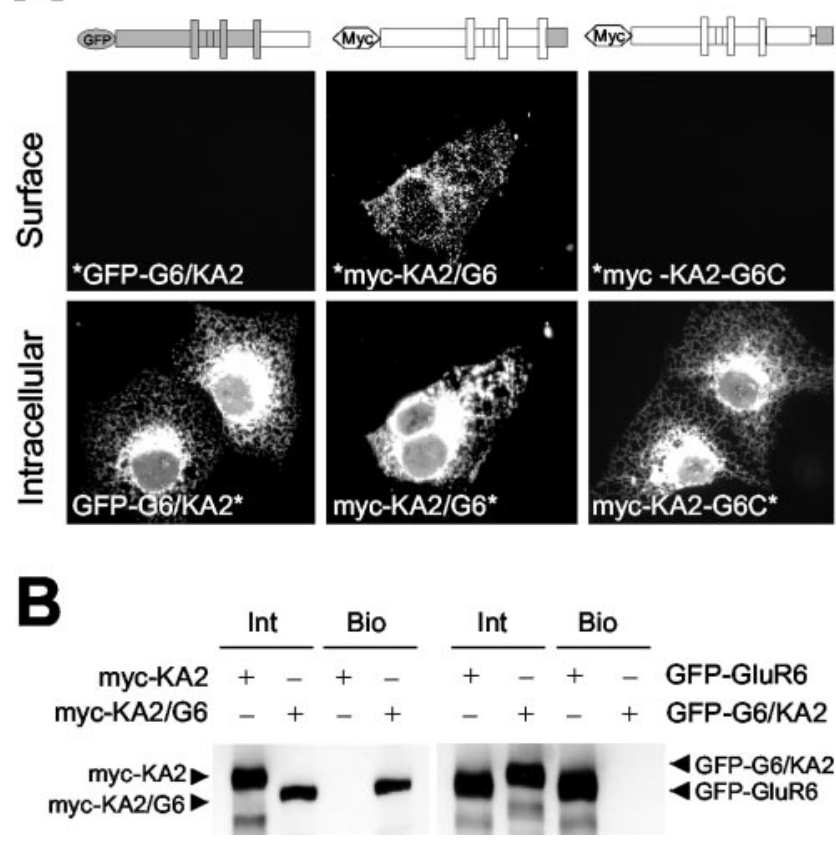

Figure 4. The C terminus of KA2 contains a signal sufficient and necessary for intracellular retention. $A$, Surface and intracellular expression of GluR6 and KA2 chimeras with swapped $C$ termini, GFP-GluR6/KA2 (left) and myc-KA2/GluR6 (middle). Also shown is the surface and intracellular expression of another KA2 chimera with an appended GluR6 ( terminus (right). Live cells were labeled with anti-AFP or anti-myc antibodies (Surface), permeabilized, and labeled with anti-KA2 or anti-GluR6 antibodies (Intracellular). The constructs used for the staining are illustrated at the top; white bars correspond to KA2 sequences, and gray bars correspond to GluR6 sequences. Asterisks indicate the antibodies used. $B$, Surface expression of wild-type and chimeric GluR6 and KA2 receptors evaluated by biotinylation. Transfected live HEK293 cells were surface-biotinylated with sulfo-NHS-S-S-biotin, and the biotinylated (Bio) and intracellu$\operatorname{lar}(\operatorname{Int})$ fractions of the cell extract were purified and immunoblotted with anti-myc or anti-GFP antibodies. Myc-KA2 (left panel, third lane) and GFP-GluR6/KA2 (right panel, fourth lane) are not present in the biotinylated fractions, whereas myc-KA2/G6 (left panel, fourth lane) and GFP-GluR6 (right panel, third lane) are detected. line. KA2 constructs tagged with either HA or FLAG epitopes at the extreme C termini (named KA2-HA and KA2-FLAG, respectively) were generated (Fig. $1 A$ ). When cotransfected into HEK293 cells, both tagged KA2 subunits were expressed well and were incorporated into KA2 homomeric complexes (Fig. 1B). This clearly indicates that KA2 subunits are capable of forming homomultimeric (i.e., at least homodimeric) receptors, although the precise stoichiometry remains to be defined.

Retention of homomeric KA2 receptors in intracellular compartments may also explain their lack of channel activity. To investigate whether heterologously expressed KA2 homomeric receptors are expressed on the plasma membrane, we inserted a tag (consisting of five myc epitopes in tandem) into the $\mathrm{N}$ terminus of KA2. The myc-KA2 fusion protein behaves the same way as the wild-type subunit, evident by its ability to form functional heteromeric receptors with GluR5 (see Fig. 6) and GluR6 subunits (see Fig. $7 B$ ). We then transfected the myc-KA2 into COS-7 cells and used a live immunofluorescence method to visualize cell surfaceexpressed receptors. No surface expression of KA2 could be detected (Fig. 2, left panels), even after repeated attempts in several different cell lines (data not shown). Instead, permeabilized staining revealed that KA2 receptors were localized to a mesh network-like intracellular compartment reminiscent of ER morphology, with most of the staining concentrated at a perinuclear region. In contrast, GFPtagged GluR6 was detected on the plasma membrane with the same staining protocol (Fig. 2, right panels).

To further confirm the intracellular retention of KA2, we performed a surface biotinylation experiment. The membraneimpermeable sulfo-NHS-S-S-biotin bound covalently to surface proteins, which were then separated from the intracellular pools using streptavidin beads. Once again, we found that KA2 homomeric receptors were not expressed on the plasma membrane but, rather, were retained intracellularly (see Fig. $4 B$, left panel) In contrast, GluR6 homomeric receptors were readily detected on the cell surface (see Fig. $4 B$, right panel), in agreement with the staining results.

The two major intracellular organelles involved in protein biogenesis and trafficking are the ER and Golgi apparatus, both of which have distinctive morphology and unique molecular markers. To determine the precise intracellular location of the KA2 homomeric receptors, we compared their distribution profile with those of the ER and Golgi markers. When expressed in COS-7 or NRK cells, KA2 colocalized extensively with the ER markers calnexin and DsRed-ER (Fig. $3 A$ ) but showed no overlap with Golgi proteins giantin, mannosidase II, and TGN-38 (Fig. 3B).

Together these data suggest that the inability of KA2 to form functional channels is not attributable to the lack of subunit oligomerization but, rather, is determined by its ER retention and failed delivery to the plasma membrane.

\section{The $\mathrm{C}$ terminus of KA2 contains an arginine-rich ER} retention/retrieval signal and a di-leucine endocytic motif Because ER retention signals have been identified in the cytoplasmic C-terminal tail of the NMDA receptor subunit NR1, as well 
as many other transmembrane proteins, we postulated that the KA2 subunit might also contain ER retention signals in its $\mathrm{C}$ terminus. To test this hypothesis, we generated chimeric receptors by exchanging the C-terminal domains of GluR6 and KA2 subunits. The resulting chimeras, GFP-GluR6/KA2 (GFP-tagged GluR6 with the $\mathrm{C}$ terminus of $\mathrm{KA} 2$ ) and mycKA2/G6 (myc-tagged KA2 with the C terminus of GluR6), were then used to examine whether the C-terminal domains controlled the trafficking profiles of both subunits. When expressed in COS-7 cells, GFP-GluR6/KA2 showed an intracellular distribution pattern similar to that of the wild-type KA2, with no detectable staining on the plasma membrane (Fig. $4 A$, left panels), whereas myc-KA2/G6 exited the ER and was readily detected on the cell surface (Fig. $4 A$, middle panels). In addition, simply attaching the GluR6 $\mathrm{C}$ terminus to KA2 did not promote its surface expression (Fig. $4 A$, right panels). Similar results were also obtained by surface biotinylation experiments (Fig. $4 B$ ). These data indicate that the KA2 $\mathrm{C}$ terminus contains a trafficking signal(s) that is necessary and sufficient to confer ER retention.

To facilitate the search for the precise ER retention signal in the KA2 C-terminal region and to avoid the complication of heteromeric assembly with native subunits in neurons, we constructed a reporter molecule, the Tac-KA2 chimera. It consists of the N-terminal and transmembrane domains of the human interleukin 2 receptor $\alpha$ subunit (Tac) and the $\mathrm{C}$ terminus of KA2 (Fig. 5A). Initial experiments in both COS-7 cells and cultured hippocampal neurons confirmed that, although the wild-type Tac showed strong surface expression (Fig. 5B, C, first panels), the Tac-KA2 chimera was intracellularly retained with no detectable surface staining (Fig. 5B, $C$, second panels). Similar results were obtained by using flow assisted cytometry (FAC) to quantify surface expression (Fig. 5D). These results not only validate the TacKA2 chimera as a legitimate substitute for KA2 in the ensuing mutational analysis but also demonstrate the universal nature of the putative ER retention/retrieval signal, which remains functional even in the context of an unrelated protein.

The KA2 C-terminal domain contains several sequences that are potential trafficking signals, including an arginine-rich motif (RRRRR862-866) similar to the aforementioned RXR-type ER retention signal and two di-leucine motifs (LL874-875 and LL908-909), which have potential roles in clathrin-dependent endocytosis (Letourneur and Klausner, 1992). Deletion of the last 113 amino acids (containing both di-leucine motifs) from TacKA2 did not affect its ER retention (Fig. 5B,C, third panels), whereas deletion of the last 118 residues (also containing argi-
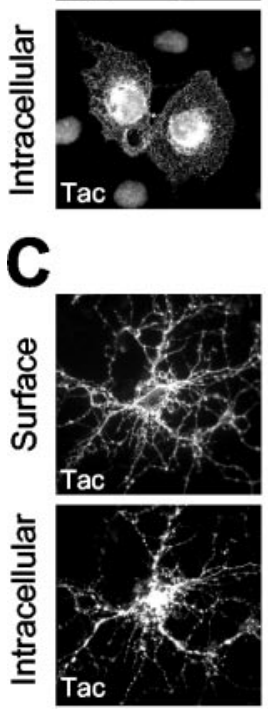
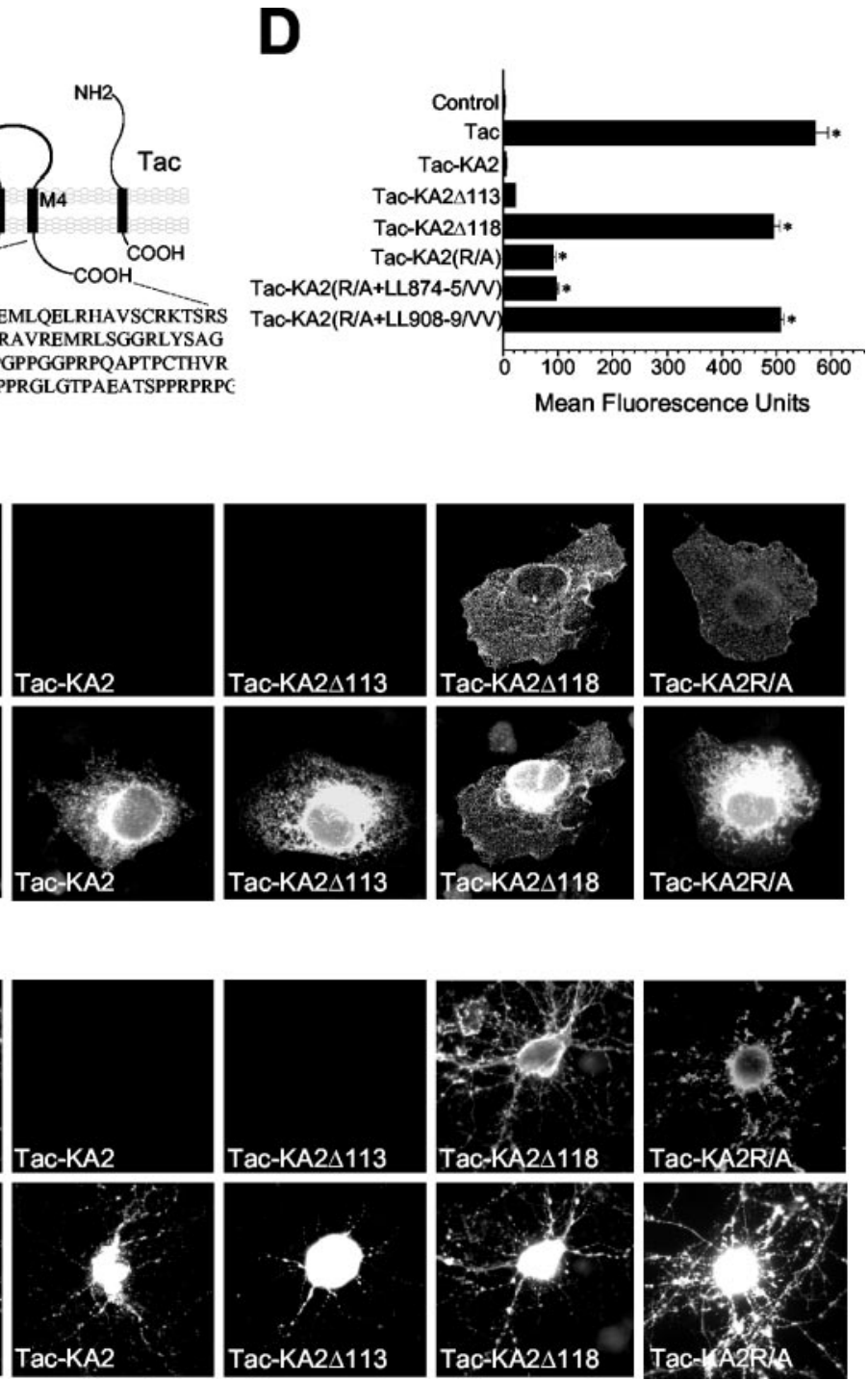

Figure 5. Identification of an arginine-rich motif and a di-leucine sequence that mediate intracellular retention of KA2. $A$, Schematic diagram illustrating the structures of KA2 and Tac. M1-M4 indicate the four membrane-spanning segments. The KA2 a single transmembrane domain with a long $\mathrm{N}$-terminal region and a short C-terminal tail. $\mathrm{COOH}, \mathrm{C}$ terminus; $\mathrm{NH} 2, \mathrm{~N}$ terminus. $B$ Surface and intracellular expression of Tac and TacKA2 mutants in COS cells. Tac and Tac-KA2 mutants containing deletion or point

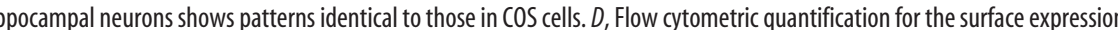
whereas additional mutation (LL908 -909VV) leads to full restoration of the surface expression. Data represent means \pm SEM of fluorescence intensities from 50,000 transfected cells stained for surface expression of Tac or TacKA2 chimeras. ${ }^{*} p<0.05$ relative to Tac minus primary antibody control, Student's unpaired $t$ test.

nines 862-866) led to strong surface expression (Fig. 5B,C, fourth panels). These results suggest that the arginine-rich motif serves as an ER retention signal. In support of this idea, we also found that another Tac-KA2 mutant (named Tac-KA2R/A), in which arginines $862-866$ were mutated to alanines, was readily immunostained on the cell surface (Fig. 5B, C, fifth panels). However, quantification data showed that Tac-KA2R/A had a much lower surface expression level compared with the wild-type Tac and Tac-KA2 $\Delta 118$ mutant (Fig. $5 D$ ). This is unlikely to be a result of differential expression because all proteins are expressed at comparable levels, evident from the intracellular staining (Fig. $5 B, C$, bottom panels) and Western blotting (data not shown). To 
A

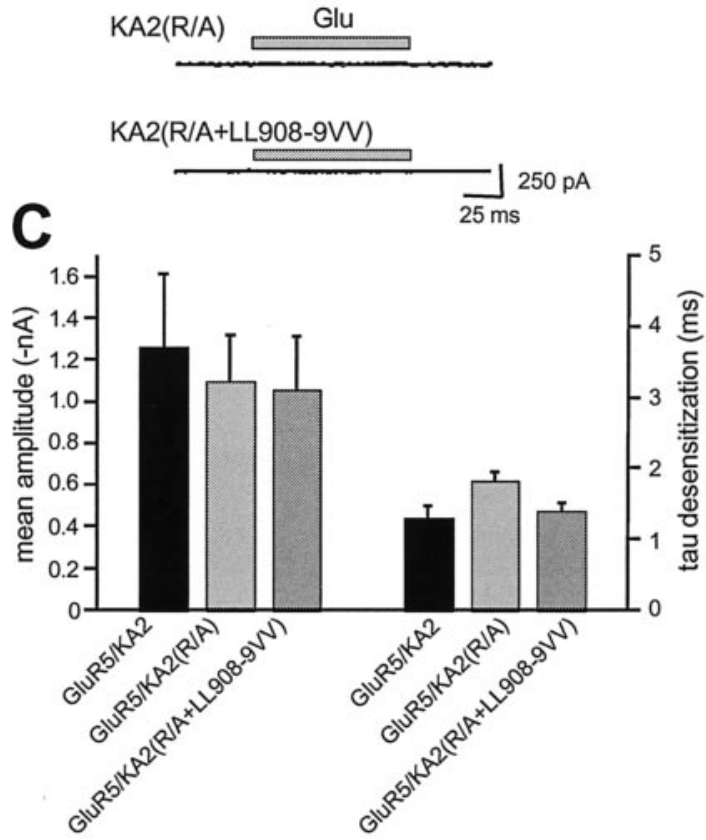

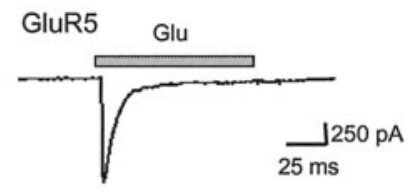

GluR5/KA2

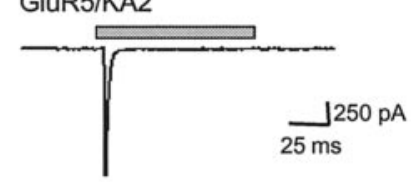

GluR5/KA2(R/A)

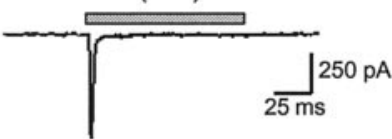

GluR5/KA2(R/A+LL908-9VV)

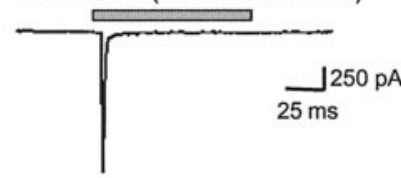

vent the surface expression of nonfunctional receptors but only allow the delivery of functional heteromeric receptors to the plasma membrane.

\section{Heteromeric assembly sterically masks} the ER retention/retrieval signal

Because KA2-containing heteromeric receptors are expressed on the plasma membrane, mechanisms must exist to occlude the ER retention/retrieval signal. One possibility is that the presence of dominant forward-trafficking signals in GluR5-7, which can overpower the retention signal in KA2. Alternatively, the retention signal may be masked because of steric hindrance or specific interaction among the $\mathrm{C}$ ter$\mathrm{mini}$, as reported for other heteromeric receptors (Margeta-Mitrovic et al., 2000; Keller et al., 2001). The first scheme is unlikely, because the GFP-GluR6/KA2 chimera was retained in the ER just like KA2 (Fig. 4A, left panels). Furthermore, appending the GluR6 C-terminal sequence to KA2 did not affect its ER retention (Fig. $4 A$, right panels). These data effectively eliminate the existence of dominant forward-trafficking signals in GluR6.

We then focused on the latter hypothesis, exploring how this ER retention/retrieval signal might be shielded in a heteromeric receptor complex. A GFP fusion protein of the GluR6 C terminus was constructed to examine whether it could bind directly to KA2, thus masking the reten-

explain the decreased surface expression of TacKA2(R/A) mutant, we examined the potential role of each di-leucine motif, using constructs that contain point mutations at arginines 862866 and either of the two di-leucine sites. The additional mutation of LL874-875 to valines had no effect on the surface expression, whereas a similar change at LL908-909 fully restored the expression level. This finding is consistent with a scenario that the di-leucine motif (i.e., LL908-909) mediates the internalization of surface-expressed TacKA2R/A mutant and its subsequent relocalization to the intracellular compartments.

\section{Surface-expressed homomeric KA2 receptors remain nonfunctional}

We next tested whether KA2 mutants with disrupted ER retention signals [i.e., myc-KA2(R/A) and myc-KA2(R/A+LL908-9VV) mutants] could form functional channels. When expressed as homomeric receptors, both myc-KA2(R/A) and mycKA2(R/A+ LL908-9VV) remained nonresponsive to glutamate (Fig. 6A) despite robust surface expression (Fig. $7 B$ ). Pretreatment of receptor-expressing cells with $0.3 \mathrm{mg} / \mathrm{ml}$ concanavalin $\mathrm{A}$, which potentiates steady-state kainate receptor currents, did not yield detectable currents during glutamate application (data not shown). When coexpressed with GluR5-2a subunits, the GluR5/ KA2(R/A) and GluR5/KA2(R/A+LL908-9VV) receptors gave glutamate with amplitude and kinetics similar to those of the wild-type GluR5/KA2 receptors (Fig. 6B,C), suggesting that the mutations in KA2 did not affect its heteromeric assembly. Together these data reaffirm the notion that trafficking checkpoints are in place to pre- tion signal. Coimmunoprecipitation experiments showed no interaction between them, and overexpression of this fusion protein did not promote KA2 egress from the ER (data not shown). These data suggest that the signal-masking process is unlikely because of specific interaction among the $\mathrm{C}$ termini of constituent subunits but, rather, is mediated through nonspecific steric hindrance. To further test this idea, we constructed a GFPGluR6/1 chimera, in which the C terminus of GluR6 was replaced with that of AMPA receptor subunit GluR1. Despite the minimal sequence homology between the two C-terminal domains (but similarity in length), the GluR6/1 chimera effectively assembled with myc-KA2 (Fig. 7A) and enabled the heteromeric receptors to pass through the secretory pathway onto the plasma membrane (Fig. $7 B$ ). These results suggest that steric hindrance may play a key role in masking the ER retention/retrieval signal during heteromeric assembly.

\section{Discussion}

The controlled surface expression of ionotropic glutamate receptors plays a key role in modifying the strength of excitatory synapses. In particular, the insertion and removal of AMPA receptors from synapses may mediate the postsynaptic induction of long-term potentiation and long-term depression, respectively (for review, see Barry and Ziff, 2002). Other studies demonstrate that trafficking signals also exist to control surface expression of NMDA receptors (Standley et al., 2000; Scott et al., 2001). In contrast, the mechanisms regulating surface expression of kainate receptors remain unknown. Physiological studies demon- 
A

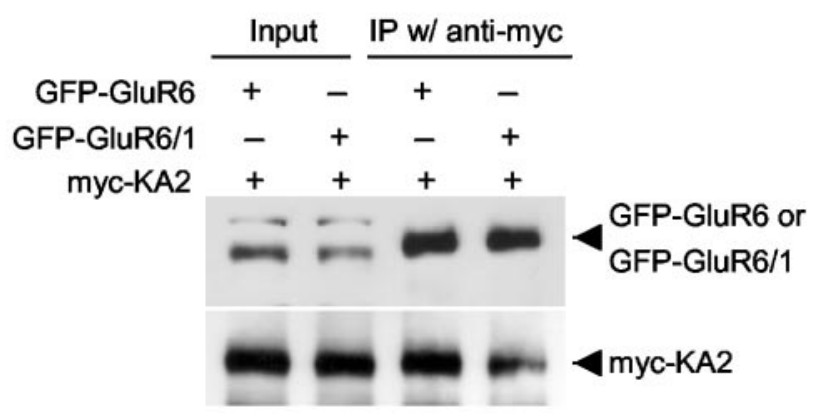

B

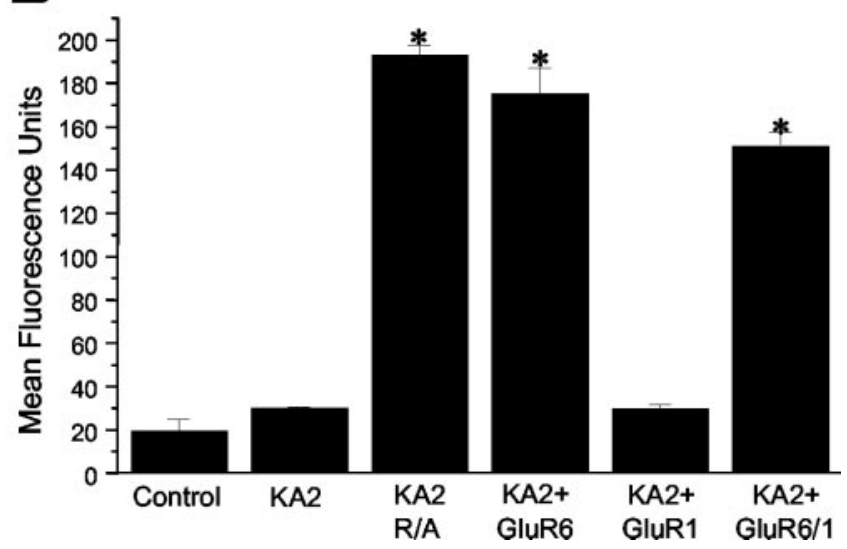

Figure 7. The ER retention/retrieval signal is sterically masked by heteromeric assembly. $A$, Both GluR6 and GluR6/1 chimeras can assemble with KA2 in heterologous cells. HEK293 cells were cotransfected with myc-KA2 and GFP-GluR6 (or GFP-GluR6/1 chimera) as indicated. The cell lysate was immunoprecipitated (IP w/) using anti-myc antibody, and the precipitates were immunoblotted with anti-myc or anti-GFP antibodies. B, Surface expression of a KA2 mutant with a disrupted ER retention signal and heteromerically assembled wild-type KA2 shown by FAC quantification. HEK293 cells were transfected with myc-KA2 [or myc-KA2(R/A) mutant] alone or together with other subunits (GluR6, GluR1, or GluR6/1) as indicated. Data represent means \pm SEM of fluorescence intensities from 50,000 transfected cells stained for surface expression of KA2. ${ }^{*} p<0.05$ relative to KA2 minus primary antibody control, Student's unpaired $t$ test.

strate that the "high-affinity" kainate receptor subunits KA1 and KA2 do not form functional homomeric receptors (Herb et al., 1992), but the underlying molecular mechanisms have not been defined. Here, we report the identification of two trafficking signals present in the $\mathrm{C}$ terminus of KA2, namely an arginine-rich ER retention/retrieval motif and a di-leucine endocytic sequence. Disruption of the ER retention/retrieval signal allows surface expression of KA2 homomeric receptors that remain nonfunctional. We further show that, during heteromeric assembly, the ER retention/ retrieval signal is likely masked by steric hindrance, thus allowing surface expression of functional heteromeric receptors.

\section{Intracellular retention of KA2 is mediated by discrete trafficking signals}

The molecular mechanisms that ensure proper folding, assembly, and delivery of multimeric transmembrane proteins to their target compartments have been extensively studied. ER retention/ retrieval may serve as an important checkpoint, ensuring that only fully assembled functional complexes are expressed on the cell surface (Teasdale and Jackson, 1996). The unassembled subunits, misfolded subunits, or both are retained in the ER and may be further targeted for degradation by ubiquitination and cleavage in the proteosome (Keller et al., 1998, 2001).

The ER retention/retrieval process can be initiated through the recognition of discrete retention signals or protein misfolding. The best-characterized ER retention signals are the luminal $\mathrm{H} / \mathrm{KDEL}$ sequence and the cytoplasmic KKXX motif (Teasdale and Jackson, 1996). The H/KDEL sequences are found at the C termini of many soluble proteins, whereas the KKXX motifs are present at the extreme C-terminal regions of certain transmembrane proteins. Proteins that contain these signals are retained in the ER on their retrieval from the cis-Golgi or ER-Golgi intermediate compartment (Teasdale and Jackson, 1996). In a series of recent reports, a novel RXR-type ER retention signal has been characterized in several transmembrane proteins (Zerangue et al., 1999; Margeta-Mitrovic et al., 2000; Standley et al., 2000; Scott et al., 2001). This motif functions in yeast, Xenopus oocytes, and mammalian cells, suggesting that it is evolutionarily conserved and can be recognized by the eukaryotic trafficking machinery (Zerangue et al., 1999).

In this study, we demonstrated, by domain swapping, that intracellular retention signals existed in the C-terminal region of KA2. The exact motifs were then identified through mutation analysis of a Tac-KA2 chimera. This approach has been used extensively for the identification of trafficking signals in the secretory pathway (Dittrich et al., 1996; Tan et al., 1998; Fu and Kreibich, 2000; Aguilar et al., 2001) because of the monomeric nature of the Tac protein and the existence of highly specific N-terminal antibodies.

Two mechanisms, which use distinct cellular machineries to control intercompartmental traffic along the secretory pathway, are shown to mediate the intracellular retention of KA2. Together they represent a coordinated quality control mechanism that ensures that only fully assembled functional receptors are expressed on the cell surface. The first mechanism regulates ER retention/ retrieval and is operated through the recognition of the argininerich motif. Clearly this arginine-rich motif differs from the classic KKXX and H/KDEL signals in both its amino acid requirement and its proximal location in the $\mathrm{C}$ terminus. However, it is not entirely clear whether it belongs to the aforementioned RXR family of ER retention/retrieval signals, despite their similar residue composition. Further studies are necessary to examine whether it shares other reported characteristics, in particular the location independence feature proposed as a hallmark for the RXR-type signals (Zerangue et al., 1999). What also remains undefined is the precise cellular machinery involved in this poly-argininemediated ER retention/retrieval. Previous studies have revealed that the $\mathrm{H} / \mathrm{KDEL}$ - or KKXX-containing proteins use coatomer complex I as their retrograde-trafficking vehicle (Teasdale and Jackson, 1996). Whether the poly-arginine-mediated event resembles this scenario or uses its own unique mechanism is open to investigation.

A di-leucine motif serves as the second checkpoint preventing inappropriate surface expression. The KA2 subunits that escaped the ER retention can be retrieved from the plasma membrane or the TGN back to endosomes or further to the lysosomes for degradation. Adapter protein complexes are believed to recognize the di-leucine sequence and to initiate these clathrin-dependent trafficking processes (Teasdale and Jackson, 1996). The fact that only one of the two di-leucine motifs seems functional is not entirely surprising, because this motif is well documented to act in a context-dependent manner (for review, see Kirchhausen, 1999). The di-leucine motif in KA2 is the first endocytic signal identified in kainate receptor subunits. Although this study has 
not addressed its functional significance in KA2-containing heteromeric receptors, it is conceivable that this di-leucine motif may regulate the dynamic turnover of these receptors at the cell surface, similar to the clathrin-dependent endocytosis reported for AMPA receptors (for review, see Barry and Ziff, 2002).

The arginine-rich ER retention/retrieval signal, although functioning in the KA2 homomeric receptors, is disabled in the KA2-containing heteromeric complexes. Its occlusion on heteromeric assembly is likely mediated through steric hindrance, rather than specific interaction among the $\mathrm{C}$ termini of constituent subunits. In support of this idea, replacing the $\mathrm{C}$ terminus of GluR6 with that of GluR1 does not impair its ability to occlude the ER retention/ retrieval signal. Although the precise molecular determinant remains unclear, one or both of the following mechanisms are likely to be involved. One possibility is that the signal-masking process relies on the steric hindrance provided by the $\mathrm{C}$ termini of assembling partners (i.e., GluR5/6/7). The other scenario is that some conformational changes acquired by KA2 during heteromeric assembly are sufficient to complete this maneuver.

Finally, it is interesting to point out that there is a similar arginine-rich motif in the $\mathrm{C}$ terminus of the KAl subunit. It is tempting to propose that KA1 may also be retained in the ER through a similar mechanism, given its functional resemblance to KA2. Indeed, our preliminary experiments suggest that mutating the arginine-rich sequence in KA1 promote its surface expression.

\section{Physiological implications of the trafficking signals in kainate receptor function}

The existence of an arginine-rich ER retention/retrieval signal and a di-leucine endocytic motif in KA2 raises the interesting possibility that these signals may control the supply and removal of KA2-containing kainate receptors to and from the plasma membrane, thus regulating the number of these receptors on the cell surface. The KA2-assembling partners (i.e., GluR5/6/7) share high overall homology ( $\sim 75-80 \%)$, but significant discrepancies still exist in individual domains, such as the distinct $\mathrm{C}$ termini of GluR5 splice variants. These differences may affect their ability to mask the trafficking signals present in KA2, thus providing a mechanism that enables differential surface expression of heteromeric kainate receptors with distinct subunit compositions. In support of this idea, it has been shown that the RXR motif in the $\mathrm{K}_{\mathrm{ATP}}$ channel subunit sulfonylurea receptor is partially exposed (i.e., may weakly interact with the retention machinery) in the fully assembled heterooctameric channel (Zerangue et al., 1999). Further study of this mechanism may provide valuable insights toward understanding the functional diversity of kainate receptors.

The RXR-mediated ER retention of NR1 can be modulated by protein phosphorylation and interaction with PDZ (postsynaptic density-95/Discs large/zona occludens-1)-containing proteins (Standley et al., 2000; Scott et al., 2001). These findings add another level of regulation regarding receptor assembly and trafficking. Because consensus phosphorylation sites in KA2 are near the arginine-rich motif, and KA2 is also known to interact with the synapse-associated protein (SAP) 90/PSD-95 family of scaffolding proteins through its C-terminal PXXP motifs (Garcia et al., 1998), we examined their potential roles in KA2 trafficking. Phosphorylation-mimicking mutation of the serine and threonine residues in TacKA2 [i.e., Tac-KA2(TS/DD)] did not promote its egress from the ER (Fig. 8A). Coexpression of SAP90 with KA2 also had no effect on its ER retention (Fig. $8 B$ ). These discrepancies regarding KA2 and NR1 subunits indicate that either the two poly-arginine signals are different despite their sim-

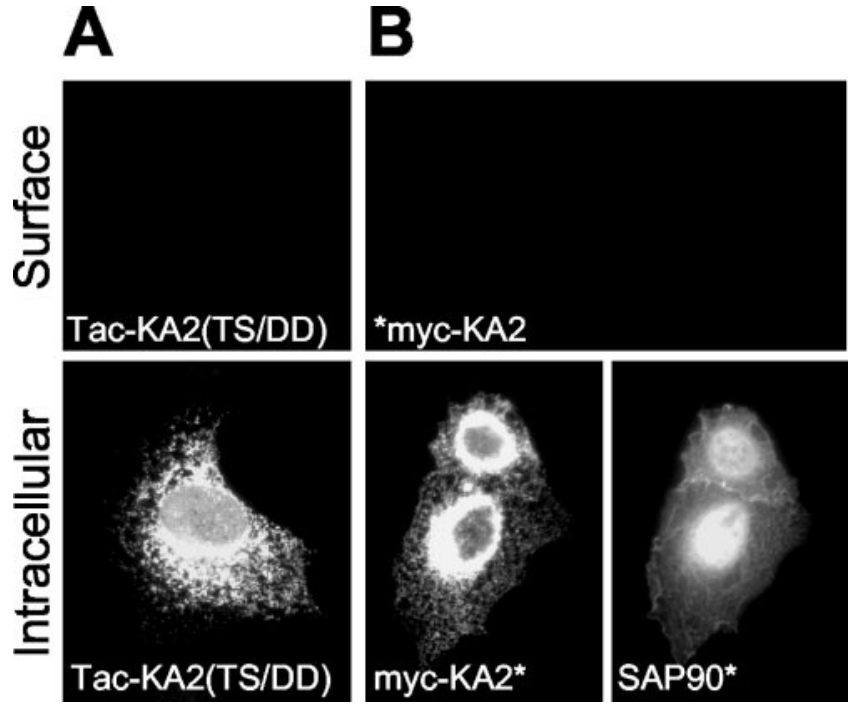

Figure 8. Protein phosphorylation and a KA2-interacting protein do not regulate the ER retention of KA2. A, Surface (top) and intracellular (bottom) expression of TacKA2 (T858S859$D D)$, a phosphorylation-mimicking mutant. $B$, Surface (top) and intracellular (bottom left) expression of KA2, as well as intracellular expression of cotransfected SAP90 (bottom right). Note that there is no surface staining for either of the receptors.

ilar residue composition, or they are simply regulated by distinct mechanisms. In fact, mounting evidence suggests that kainate receptors may use unique trafficking mechanisms different from those used by other ionotropic glutamate receptors. For instance, it has been shown that certain synaptic contacts only contain kainate receptors (DeVries and Schwartz, 1999). Moreover, within a single neuron, kainate receptors can be targeted to specific synaptic terminals, with no colocalization with NMDA or AMPA receptors (for review, see Lerma et al., 2001). Clearly, future studies beyond the scope of this report are necessary to address all these issues. Nonetheless, they further highlight the extraordinary complexity of the molecular mechanisms regulating intracellular trafficking, subunit assembly, and surface expression of kainate receptors that we are just beginning to understand.

\section{References}

Aguilar RC, Boehm M, Gorshkova I, Crouch RJ, Tomita K, Saito T, Ohno H, Bonifacino JS (2001) Signal-binding specificity of the mu4 subunit of the adaptor protein co mplex AP-4. J Biol Chem 276:13145-13152.

Barry MF, ZiffEB (2002) Receptor trafficking and the plasticity of excitatory synapses. Curr Opin Neurobiol 12:279-286.

Bettler B, Boulter J, Hermans-Borgmeyer I, O'Shea-Greenfield A, Deneris ES, Moll C, Borgmeyer U, Hollmann M, Heinemann SF (1990) Cloning of a novel glutamate receptor subunit, GluR5: expression in the nervous system during development. Neuron 5:583-595.

Bowie D, Lange GD (2002) Functional stoichiometry of glutamate receptor desensitization. J Neurosci 22:3392-3403.

Cui C, Mayer ML (1999) Heteromeric kainate receptors formed by the coassembly of GluR5, GluR6, and GluR7. J Neurosci 19:8281-8291.

DeVries SH, Schwartz EA (1999) Kainate receptors mediate synaptic transmission between cones and "Off" bipolar cells in a mammalian retina. Nature 397:157-160.

Dittrich E, Haft CR, Muys L, Heinrich PC, Graeve L (1996) A di-leucine motif and an upstream serine in the interleukin-6 (IL-6) signal transducer gp130 mediate ligand-induced endocytosis and down-regulation of the IL-6 receptor. J Biol Chem 271:5487-5494.

Fu J, Kreibich G (2000) Retention of subunits of the oligosaccharyltransferase complex in the endoplasmic reticulum. J Biol Chem 275:3984-3990.

Garcia EP, Mehta S, Blair LA, Wells DG, Shang J, Fukushima T, Fallon JR, 
Garner CC, Marshall J (1998) SAP90 binds and clusters kainate receptors causing incomplete desensitization. Neuron 21:727-739.

Herb A, Burnashev N, Werner P, Sakmann B, Wisden W, Seeburg PH (1992) The KA-2 subunit of excitatory amino acid receptors shows widespread expression in brain and forms ion channels with distantly related subunits. Neuron 8:775-785.

Hollmann M, Heinemann SF (1994) Cloned glutamate receptors. Annu Rev Neurosci 17:31-108.

Keller SH, Lindstrom J, Taylor P (1998) Inhibition of glucose trimming with castanospermine reduces calnexin association and promotes proteasome degradation of the $\alpha$-subunit of the nicotinic acetylcholine receptor. J Biol Chem 273:17064-17072.

Keller SH, Lindstrom J, Ellisman M, Taylor P (2001) Adjacent basic amino acid residues recognized by the COP I complex and ubiquitination govern endoplasmic reticulum to cell surface trafficking of the nicotinic acetylcholine receptor $\alpha$-subunit. J Biol Chem 276:18384-18391.

Kirchhausen T (1999) Adaptors for clathrin-mediated traffic. Annu Rev Cell Dev Biol 15:705-732.

Kowalski JM, Parekh RN, Mao, Wittrup KD (1998) Protein folding stability can determine the efficiency of escape from endoplasmic reticulum quality control. J Biol Chem 273:19453-19458.

Laube B, Kuhse J, Betz H (1998) Evidence for a tetrameric structure of recombinant NMDA receptors. J Neurosci 18: 2954-2961.

Lerma J, Paternain AV, Rodriguez-Moreno A, Lopez-Garcia JC (2001) Molecular physiology of kainate receptors. Physiol Rev 81:971-998.

Letourneur F, Klausner RD (1992) A novel di-leucine motif and a tyrosinebased motif independently mediate lysosomal targeting and endocytosis of CD3 chains. Cell 69:1143-1157.

Letourneur F, Hennecke S, Demolliere C, Cosson P (1995) Steric masking of a di-lysine endoplasmic reticulum retention motif during of the human high affinity receptor for immunoglobulin E. J Cell Biol 129:971-978.

Ma D, Zerangue N, Lin YF, Collins A, Yu M, Jan YN, Jan LY (2001) Role of ER export signals in controlling surface potassium channel numbers. Science 291:316-319.

Margeta-Mitrovic M, Jan YN, Jan LY (2000) A trafficking checkpoint controls GABA(B) receptor heterodimerization. Neuron 27:97-106.

Paternain AV, Herrera MT, Nieto MA, Lerma J (2000) GluR5 and GluR6 kainate receptor subunits coexist in hippocampal neurons and coassemble to form functional receptors. J Neurosci 20:196-205.

Rosenmund C, Stern-Bach Y, Stevens CF (1998) The tetrameric structure of a glutamate receptor channel. Science 280:1596-1599.

Schiffer HH, Swanson GT, Heinemann SF (1997) Rat GluR7 and a carboxyterminal splice variant, GluR7b, are functional kainate receptor subunits with a low sensitivity to glutamate. Neuron 19:1141-1146.

Scott DB, Blanpied TA, Swanson GT, Zhang C, Ehlers MD (2001) An NMDA receptor ER retention signal regulated by phosphorylation and alternative splicing. J Neurosci 21:3063-3072.

Seeburg PH (1993) The molecular biology of mammalian glutamate receptor channels. Trends Neurosci 16:359-365.

Standley S, Roche KW, McCallum J, Sans N, Wenthold RJ (2000) PDZ domain suppression of an ER retention signal in NMDA receptor NR1 splice variants. Neuron 28:887-898.

Stockklausner C, Ludwig J, Ruppersberg JP, Klocker N (2001) A sequence motif responsible for ER export and surface expression of Kir2.0 inward rectifier $\mathrm{K}(+)$ channels. FEBS Lett 493:129-133.

Swanson GT, Gereau RW, Green T, Heinemann SF (1997) Identification of amino acid residues that control functional behavior in GluR5 and GluR6 kainate receptors. Neuron 19:913-926.

Tan PK, Waites C, Liu Y, Krantz DE, Edwards RH (1998) A leucine-based motif mediates the endocytosis of vesicular monoamine and acetylcholine transporters. J Biol Chem 273:17351-17360.

Teasdale RD, Jackson MR (1996) Signal-mediated sorting of membrane proteins between the endoplasmic reticulum and the Golgi apparatus. Annu Rev Cell Dev Biol 12:27-54.

Wenthold RJ, Trunpy VA, Zhu WS, Petralia RS (1994) Biochemical and assembly properties of GluR6 and KA2, two members of the kainate receptor family, determined with subunit-specific antibodies. J Biol Chem 269:1332-1339.

Xia H, Von Zastrow M, Malenka RC (2002) A novel anterograde trafficking signal present in the N-terminal extracellular domain of ionotropic glutamate receptors. J Biol Chem 277:47765-47769.

Zerangue N, Schwappach B, Jan YN, Jan LY (1999) A new ER trafficking signal regulates the subunit stoichiometry of plasma membrane K(ATP) channels. Neuron 22:537-548. 